\title{
ANALYTIC SINGULAR INTEGRAL OPERATORS ${ }^{1}$
}

\author{
BY WILLIAM MARGULIES
}

Communicated by A. P. Calderon, January 11, 1967

The following paper extends to real analytic manifolds the general theory of singular integral operators as described in [10] and [13].

The definition of an analytic singular integral operator is made in terms of the kernel of the operator. The symbol of the operator is discussed and in the case of an elliptic operator, a regularity theorem is proved.

It should be pointed out, however, that the regularity theorem is a purely local one. The question of obtaining a global inverse to an elliptic operator or more generally an operator with a prescribed symbol is still open.

1. Definition and coordinate invariance. We recall the definition of a modified homogeneous distribution of degree $\lambda$.

(i) If $\lambda$ is not a positive integer, then a modified homogeneous distribution of degree $\lambda$ is a homogeneous distribution of degree $\lambda$.

(ii) If $\lambda$ is a positive integer $\geqq 0$, then $f$ is a modified homogeneous distribution of degree $\lambda$ iff $f=g_{\lambda}+P_{\lambda}(x) \log |x|$, where $g_{\lambda}$ is orthogonal to all polynomials homogeneous of degree $\lambda$ and $P_{\lambda}(x)$ is a homogeneous polynomial of degree $\lambda$.

Let $M$ be a compact, real analytic manifold without boundary of $\operatorname{dim} \nu$.

Definition 1.1. $A$ is an analytic singular integral operator of order $\lambda$ iff

(i) the kernel of $A$ is analytic off the diagonal in $M \times M$;

(ii) for each $\Psi$, a coordinate function, and $p$ in the domain of $\Psi$ with $\Psi(p)=x_{0}$, there is an $\epsilon>0$ such that

$$
A_{x}=\sum_{i \geq 0} A_{x}^{i-\lambda-\nu}+C_{x}
$$

where $A_{x}^{i-\lambda-\nu}$ is a modified homogeneous distribution of degree $i-\lambda-\nu$ with a kernel

$$
A_{i-\lambda \rightarrow \nu}(x, z)
$$

satisfying

1 I would like to thank Dr. R. T. Seeley of Brandeis University for the helpful conversations on the material presented in this thesis. 


$$
\sup _{\substack{y \in\left\{\left|x-x_{0}\right|<e\right\} \\ z \in S^{p-1}}}\left|D_{y}^{\alpha} D_{z}^{\beta} A_{i-\lambda-\nu}(y, z)\right| \leqq \alpha ! \beta ! K^{i+|\alpha|+|\beta|+1} .
$$

$K$ is a constant independent of $i, \alpha, \beta$, and $S^{\nu-1}$ is the unit sphere in $R^{p} . C_{x}$ in (1) has a kernel $C(x, z)$ jointly analytic for $\left|x-x_{0}\right| \leqq \epsilon$ and $|z| \leqq \epsilon$.

Note. Condition (2) above implies that the kernel of (1) converges uniformly to an analytic function for $\left|x-x_{0}\right| \leqq \epsilon$ and $\epsilon / \nu \leqq|z| \leqq \epsilon$ if $\epsilon$ is small enough.

This definition is seen to be highly dependent on the particular coordinate system used. The first theorem proved is therefore:

Theorem 1.1. Definition 1.1 is invariant under real analytic nonsingular coordinate changes.

One is quite confident that the form of this definition is invariant under coordinate changes from the results in the $C^{\infty}$ case. The estimate appearing in (2) is the crucial fact to be proved.

2. Preservation of analytic functions. We prove here the

THEOREM 2.1. If $F$ is analytic and $A$ is an analytic singular integral operator, then $A F$ is analytic.

3. Symbol. The symbol of an analytic singular integral operator is defined by the formal sum

$$
\sigma(A)(x, \xi)=\sum_{i \geq 0} \sigma\left(A_{i-\lambda-\nu}\right)(x, \xi)
$$

where

$$
\sigma\left(A_{i-\lambda-\nu}\right)(x, \xi)=A_{i-\lambda-\nu}(x, \xi) \quad \text { for }|\xi|>0
$$

and the transform is taken with respect to the second variable.

Note. $\sigma\left(A_{i-\lambda-\nu}\right)(x, \xi)$ is homogeneous of degree $\lambda-i$ in $\xi$ for $|\xi|>0$.

The main theorem proved here is that a certain estimate on the symbol is equivalent to the estimate appearing in (2).

THEOREM 3.1.

iff

$$
\sup _{\substack{\left.y \in|| x-x_{0} \mid \leq e\right\} \\ z \in S^{\nu-1}}}\left|D_{x}^{\alpha} D_{z}^{\beta} A_{i-\lambda-\nu}(x, z)\right|<\alpha ! \beta ! K^{|\alpha|+|\beta|+i+1}
$$

$$
\sup _{\substack{\boldsymbol{\nu} \in\left\{\mid x-x_{p \mid \leq \epsilon\}} \\ \xi \in S^{\nu-1}\right.}} \mid D_{x}^{\alpha} D_{\xi \sigma\left(A_{i-\lambda-\nu}\right)(x, \xi) \mid \leqq \alpha ! \beta ! i ! C^{|\alpha|+|\beta|+i+1}}^{\beta}
$$

$C$ and $K$ are constants independent of $i, \alpha, \beta$. 
4. Composition of symbols. In this section we show that if $\sigma(A)$ and $\sigma(B)$ satisfy (3), then so does $\sigma(A B)$. We also show that $\sigma\left(A^{*}\right)$ satisfies (3).

In the case of an elliptic symbol (the definition of ellipticity is that $\left.\sigma\left(A_{-\lambda-\nu}\right)(x, \xi) \neq 0\right)$ it is shown that the formal symbol inverse satisfies (3) above.

5. Composition of operators. We prove in this section that the operators of Definition 1.1 are closed under composition.

THEOREM 5.1. Let $A$ and $B$ be two analytic singular integral operators of order $\lambda_{1}$ and $\lambda_{2}$ respectively. Then $A B$ is an analytic singular integral operator of order $\lambda_{1}+\lambda_{2}$.

Note. The key to this proof lies in showing that the remainder of the operator $A B$ is a jointly analytic function.

\section{Regularity theorem.}

TheOREM 6.1. Let $A$ be an elliptic analytic singular integral operator. If $A f=g$ where $g$ is an analytic function, then $f$ is an analytic function.

\section{BIBLIOGRAPHY}

1. J. de Barros Neto, Analytic distribution kernels, Trans. Amer. Math. Soc. 100 (1961), 425-438.

2. J. de Barros Neto and F. E. Browder, The analyticity of kernels, Canad. J. Math. 13 (1961), 645-649.

3. F. E. Browder, Real analytic functions on product spaces and separate analyticity, Canad. J. Math. 13 (1961), 650-656.

4. A. P. Calderón and A. Zygmund, On singular integrals, Amer. J. Math. 78 (1956), 289-309.

5. - Singular integral operators and differential equations, Amer. J. Math. 79 (1957), 901-921.

6. L. Garding, Transformation de Fourier des distributions homogènes, Bull. Soc. Math. France 89 (1961), 381-428.

7. K. Knopp, Theory of functions, Part II, Dover Publications, New York, 1947.

8. T. Kotake and M. S. Narasimhan, Fractional powers of a linear elliptic operator, Bull. Soc. Math. France (1962).

9. C. B. Morrey and L. Nirenberg, On the analyticity of the solutions of linear elliptic systems of partial differential equations, Comm. Pure Appl. Math. 10 (1957), 271290.

10. R. Palais, Seminar on the Atiyah-Singer index theorem, Annals of Mathematics Studies, Princeton, N. J., 1965, Chapter 16.

11. R. T. Seeley, Spherical harmonics, Amer. Math. Monthly (1966).

12. - Singular integrals on compact manifolds, Amer. J. of Math. 81 (1959), 659-690.

13. R. T. Seeley, Refinement of the functional calculus of Calderon and Zygmund, Koninkl. Nederl. Akademic Van Wetenschappen No. 3, 69, 1965.

14. - Homogeneous distributions, Report TW88 Mathematisch Centrum (1962).

BRANDEIS UNIVERSITY 